\title{
Optimización del tratamiento de aguas residuales domésticas mediante la implementación del Sistema MBBR - Caylloma - Aquafil
}

\author{
Optimization of domestic wastewater treatment through the implementation of \\ the MBBR System - Caylloma - Aquafil
}

Wilyn Reyes Araujo ${ }^{1}$

Recibido: Enero 2020 - Aprobado: Junio 2020 - Publicado: Junio 2020

\begin{abstract}
RESUMEN
Caylloma es un distrito que pertenece a la región Arequipa, está ubicado al sur del Perú, a una altitud promedio de $4310 \mathrm{msnm}$. Caylloma cuenta con una planta de lodos activados con capacidad para tratar hasta $200 \mathrm{~m}^{3} / \mathrm{d}$, pero dado el incremento poblacional y las temperaturas bajas, la actividad bacterial se inhibe, lo cual no garantiza la reducción significativa de la carga orgánica ni de la carga bacterial patogénica. La investigación tiene por objetivo la optimización del funcionamiento de la planta de tratamiento de aguas residuales domésticas. Durante la investigación se desarrolló la evaluación, análisis y optimización del tratamiento de aguas residuales mediante el adicionamiento de Biocarriers a los dos tanques reactores biológicos, lo cual posibilitó que el sistema de lodos activados se transforme en un sistema "Moving Bed Biofilm Reactor" -MBBR-, que consiste en el aumento de la biomasa en los tanques reactores, lo cual permite una mayor degradación de la materia orgánica, para obtener un incremento del $40 \%$ de la capacidad total de tratamiento de la planta. Culminado el proceso de optimización actualmente se cuenta con una planta capaz de tratar un caudal promedio de $280 \mathrm{~m}^{3} / \mathrm{d}$, que cubre los requerimientos de una población aproximada de 3200 habitantes.
\end{abstract}

Palabras claves: Biocarriers; biomasa; reactor de lecho móvil; tiempo de detención hidráulica (TRH).

\begin{abstract}
Caylloma is a district that belongs to the Arequipa region, is located in southern Peru, at an average altitude of 4310 meters above sea level. Caylloma has an activated sludge plant with the capacity to treat up to $200 \mathrm{~m}^{3} / \mathrm{d}$, but given the population increase and low temperatures, bacterial activity is inhibited, which does not guarantee a significant reduction in organic load or bacterial load pathogenic. The research aims to optimize the operation of the domestic wastewater treatment plant. During the investigation, the evaluation, analysis and optimization of wastewater treatment was developed by adding Biocarriers to the two biological reactor tanks, which enabled the activated sludge system to be transformed into a "Moving Bed Biofilm Reactor" -MBBR system -, which consists of the increase of biomass in the reactor tanks, which allows a greater degradation of the organic matter, to obtain an increase of $40 \%$ of the total treatment capacity of the plant. At the end of the optimization process there is currently a plant capable of treating an average flow of $280 \mathrm{~m}^{3} / \mathrm{d}$, which covers the requirements of an approximate population of 3200 inhabitantss.
\end{abstract}

Keywords: Biocarriers; biomass; mobile bed reactor; hydraulic stop time (HRT).

\footnotetext{
${ }^{1}$ Egresado de la unidad de Post Grado de la Facultad de Geología, Metalúrgica, Minera y Geográfica de la Universidad Nacional Mayor de San Marcos. Lima, Perú. Teléfono: (511) 619-7000. E-mail: wilynra@hotmail.com
} 


\section{INTRODUCCIÓN}

El distrito de Caylloma es uno de los 20 distritos de la Provincia de Caylloma, el clima es frígido, con temperatura anual promedio de $3.1^{\circ} \mathrm{C}$, con rangos entre $14.0^{\circ} \mathrm{C}$ y $-10.7^{\circ} \mathrm{C}$. Las precipitaciones alcanzan valores entre $481 \mathrm{a}$ $926 \mathrm{~mm}$ anuales. La población proyectada al año 2019 es de 3,380 habitantes.

El Sistema de Abastecimiento de Agua tiene como fuente actual 2 manantiales ubicados en el sector denominado Tinajas, que se encuentra a unos $400 \mathrm{~m}$ del centro, y abastece al $82.4 \%$ de la población total. El agua suministrada al pueblo está bajo la administración de la municipalidad distrital. Las viviendas no cuentan con un servicio de agua potable continuo, situación que se complica con un deficiente tratamiento de aguas residuales domésticas.

El Sistema de Alcantarillado fue ejecutado entre los años 1996 y 2010 y su estado de conservación es bueno. El sistema no contaba con planta de tratamiento de aguas residuales -PTAR- por lo que las aguas residuales eran vertidas directamente al río Chonta, afluente del río Apurímac, contaminándola. Posteriormente, en un intento por dar solución al problema se construyó un tanque séptico, pero al ser este una unidad de tratamiento primario, de poca capacidad, el agua se rebalsaba y contaminaba las áreas verdes que se encuentran alrededor.

En el año 2015 se instaló una planta de tratamiento de aguas residuales del tipo compacta, capas de tratar $200 \mathrm{~m}^{3} / \mathrm{d}$, esta PTAR es propiedad de Aquafil - Water Technologies y cuenta con una capacidad de atención para una población equivalente de 2,300 habitantes.

La presente investigación tiene como propósito optimizar el tratamiento de aguas residuales domésticas, mediante la implementación del sistema MBBR, que es una tecnología basada en un proceso biológico de crecimiento continuo que brinda una alta efectividad en comparación con otros sistemas, ya que tiene como ventaja principal la reducción del espacio requerido para el diseño de los tanques reactores, es decir permite incrementar en un 40 $\%$ la capacidad total de la planta de tratamiento. También se resalta la importancia de la aplicación del sistema, dado el incremento poblacional de Caylloma, esto permitirá incrementar en un $40 \%$ el caudal de tratamiento, es decir, posibilitara tratar $280 \mathrm{~m}^{3} /$ día.

\section{MÉTODOS}

\subsection{Tipo y diseño de investigación}

Respecto a tipos de investigación, según las variables, calificó como no experimental ya que no se manipula la variable no comprobada de una manera rigurosa; según el nivel de medición y análisis de información, calificó como descriptiva; según su ubicación temporal, calificó como longitudinal, cohorte y prospectiva; según la extensión del estudio, calificó como investigación de caso; según las fuentes de información, calificó como investigación de campo. En cuanto al diseño de la investigación es del tipo exploratoria, descriptiva y correlacional.

\subsection{Unidad de análisis}

La unidad de análisis fue la Planta de Tratamiento de Aguas Residuales de origen doméstico, del tipo biológico y que usan lechos móviles.

\subsection{Selección de la muestra}

Para la selección de la muestra se consideró los siguientes criterios: Ubicación geográfica y características climatológicas; caudal de agua residual tratada en la planta de tratamiento de aguas residuales; uso de las aguas residuales de origen doméstico ya tratadas; facilidades de operación y mantenimiento existentes en la PTAR.

\subsection{Técnicas de recolección de Datos}

Se acudió a las fuentes de información primaria y secundaria. Respecto a las técnicas se utilizaron los instrumentos que se detallan a continuación.

\subsubsection{Descripción de la infraestructura de tratamiento y evaluación de calidad ex antes}

Descripción cuantitativa. La planta de tratamiento de aguas residuales de Caylloma previo a la implementación del sistema permitía tratar en promedio $200 \mathrm{~m}^{3} / \mathrm{d}(2.31 \mathrm{l} / \mathrm{s}) \mathrm{de}$ aguas residuales de origen doméstico. A continuación, se describen las características principales de estas unidades de tratamiento.

\section{A) Unidades de pre tratamiento}

Cámara de rejas. - Unidad que permite retener los sólidos gruesos, es del tipo manual y existen 2 unidades que funcionan en paralelo.

Trampa de grasa. - Unidades que permiten retener natas (aceites y grasas), es del tipo manual y existen 2 unidades que funcionan en paralelo.

\section{B) Unidades de tratamiento primario}

Tanque ecualizador. - Permite homogenizar y controlar el caudal afluente para que los procesos secundario y terciario reciban un caudal constante. Tiene una capacidad útil de $106.4 \mathrm{~m}^{3}$. En este caso, esta unidad no necesita aireación.

\section{C) Unidades de tratamiento secundario}

Tanque reactor biológico. - Es donde el agua residual se mezcla con un lodo biológico en medio aireado, es decir, en un mismo tanque se combinan: aireación, reacción y clarificación. Comprende 2 unidades que funcionan en paralelo, las dimensiones de cada una son: $3.0 \mathrm{~m}$ de diámetro, $12.0 \mathrm{~m}$ de longitud y capacidad de $100.0 \mathrm{~m}^{3} / \mathrm{d}$ cada uno. La aireación proviene de la sala de sopladores.

Sedimentador secundario. - Separa los lodos activados del licor mezcla, comprende 2 unidades que funcionan en paralelo, las dimensiones aproximadas de cada uno son: $3.0 \mathrm{~m}$ de diámetro y $2.8 \mathrm{~m}$ de altura. En el fondo tienen tolva para acumulación de lodos, parte del cual se recircula al tanque reactor biológico. 
Tanque de precipitación química. - Posibilita la precipitación de contaminantes metálicos pesados. En este se lleva a cabo tres procesos: coagulación, floculación y sedimentación. Se usa como sustancias químicas alumbre y cal que provienen de dosificadores. Existe una sola unidad de $3.0 \mathrm{~m}$ de diámetro y $2.8 \mathrm{~m}$ de altura, en el fondo tiene tolva para acumulación de lodos.

D) Unidades de tratamiento terciario

Tanque de contacto. - En esta unidad se lleva a cabo el proceso de desinfección del agua utilizando Hipoclorito de Calcio -HTH- que provienen del dosificador. Existe una sola unidad de dimensiones estimadas: $1.5 \mathrm{~m}$ de diámetro y $2.9 \mathrm{~m}$ de altura, en el fondo tienen tolva para acumulación de lodos.

E) Obras complementarias

Caseta de sopladores. - Estructura de concreto armado de dimensiones: $\operatorname{largo}=2.7 \mathrm{~m}$, ancho $=2.7 \mathrm{~m}$, altura $=$ $2.5 \mathrm{~m}$., donde existen 2 sopladores marca REPICKY, de 12 HP cada uno y motores trifásicos.

Sala de control. - Estructura de concreto armado de dimensiones: $\operatorname{largo}=4.3 \mathrm{~m}$, ancho $=3.2 \mathrm{~m}$ y altura $=2.5 \mathrm{~m}$. Existen 2 Filtros de Cuarzo, aparte de las bombas y accesorios. Estos filtros, que tienen como lecho filtrante arena de cuarzo, son unidades que permiten clarificar significativamente y reducir la carga orgánica al mínimo a fin de que las aguas puedan ser reutilizadas.

Adyacente a esta sala se encuentran los 3 dosificadores: uno para el compuesto clorado, otro para sulfato de alúmina y el tercero para cal.

Evaluación cualitativa. El monitoreo inicial, antes del incremento del caudal en $40 \%$ y antes de la colocación de los Biocarriers, se realizó el día 24 de mayo del 2015. Las estaciones de monitoreo se ubicaron a la entrada y a la salida de la planta, los resultados se plasman en la Tabla 5.

\subsubsection{Evaluación de la infraestructura de tratamiento $y$ calidad ex post}

Con la finalidad de optimizar el tratamiento se requirió previamente definir los parámetros de diseño de las unidades de tratamiento, por lo que se evaluó su operatividad, cuantitativa y cualitativa, considerando el incremento de caudal de agua cruda en un $40 \%$ y la adición de los biocarriers. En la siguiente Tabla 1 se señalan los parámetros de diseño:

Tabla 1. Parámetros de diseño de sistema MBBR

\begin{tabular}{lcc}
\hline \multicolumn{1}{c}{ Parámetro } & Unidad & Cantidad \\
\hline Caudal & $\mathrm{m}^{3} / \mathrm{d}$ & 280 \\
Numero de Módulos & unidades & 2 \\
Superficie específica relleno & $\mathrm{m}^{2} / \mathrm{m}^{3}$ & 400 \\
Carga del Sistema & $\mathrm{g} \mathrm{DBO} / \mathrm{m}^{2} \mathrm{~d}$ & 10 \\
Trh reactor & $\mathrm{h}$ & 11 \\
Carga hidráulica sedimentador & $\mathrm{m} / \mathrm{h}$ & 0.902 \\
Trh sedimentador & $\mathrm{h}$ & 1.745 \\
Carga hidraulica precipitador & $\mathrm{m} / \mathrm{h}$ & 1.88 \\
Trh tanque precipitador & $\mathrm{h}$ & 0.84 \\
\hline
\end{tabular}

Fuente. AQUAFIL

\subsubsection{Evaluación de unidades de tratamiento}

La aplicación de modelos matemáticos es bastante limitada en la mayoría de aplicaciones prácticas (Wanner \& Reichert, 1996). Los modelos empíricos, los cuales incorporan un enfoque estadístico del comportamiento de las plantas, pueden ser desarrollados si la complejidad del sistema lo permite. Para determinar los parámetros de diseño se acudió al análisis matemático, modelo AQUAFIL; el volumen de relleno de los Biocarriers se obtuvo a partir de los parámetros de diseño. De los cálculos realizados se determinó la siguiente Tabla 2:

\subsubsection{Evaluación cualitativa del efluente}

Se llevaron a cabo dos monitoreos en el efluente: el primero se realizó el 28 de junio del 2015 y el segundo el 26 de

Tabla 2. Unidades de tratamiento para el volumen de relleno de Biocarriers

\begin{tabular}{|c|c|c|c|c|c|c|}
\hline \multirow[b]{2}{*}{ Parámetros de diseño } & \multirow{2}{*}{$\begin{array}{c}\text { Reactor } \\
\text { biológico }\left({ }^{*} 2\right)\end{array}$} & \multirow{2}{*}{$\underset{\left({ }^{*} 2\right)}{\operatorname{Sedimentador}}$} & \multirow{2}{*}{$\begin{array}{l}\text { Precipitador } \\
\text { Químico }\end{array}$} & \multicolumn{2}{|c|}{ Agentes coagulantes } & \multirow{2}{*}{$\begin{array}{c}\text { Tanque de } \\
\text { contacto de } \\
\text { cloro }\end{array}$} \\
\hline & & & & Cal & $\begin{array}{l}\text { Sulfato de } \\
\text { Aluminio }\end{array}$ & \\
\hline $\begin{array}{l}\text { Volumen de relleno } \\
\text { (Biocarriers) }\end{array}$ & $9.5 \mathrm{~m}^{3}$ & -- & - & -- & - & -- \\
\hline Volumen total & $160.4 \mathrm{~m}^{3}$ & $25.45 \mathrm{~m}^{3}$ & $12.25 \mathrm{~m}^{3}$ & -- & -- & $5.88 \mathrm{~m}^{3}$ \\
\hline Oxígeno requerido & $2,186.8 \mathrm{~g} / \mathrm{h}$ & -- & -- & -- & -- & -- \\
\hline Volumen de aire requerido & $0.091 \mathrm{~m}^{3} / \mathrm{h}$ & -- & -- & -- & -- & -- \\
\hline Diámetro & -- & $3.0 \mathrm{~m}$ & $3.0 \mathrm{~m}$ & -- & -- & -- \\
\hline Capacidad del dosificador & -- & -- & -- & $4.2 \mathrm{~kg} / \mathrm{d}$ & $5.6 \mathrm{~kg} / \mathrm{d}$ & $3.08 \mathrm{~kg} / \mathrm{d}$ \\
\hline Consumo & -- & -- & -- & $7.6 \mathrm{~kg} / \mathrm{d}$ & $5.89 \mathrm{~kg} / \mathrm{d}$ & $309 \mathrm{~L} / \mathrm{d}$ \\
\hline Consumo de HTH & -- & -- & -- & - & -- & $4.41 \mathrm{~kg} / \mathrm{d}$ \\
\hline
\end{tabular}

Fuente. Elaboración propia - Superficie específica de los biocarriers $=400 \mathrm{~m}^{2} / \mathrm{m}^{3}$ 
julio del 2015. Ambos monitoreos se realizaron con el caudal incrementado a $140 \mathrm{~m}^{3} / \mathrm{d}$ para cada módulo y con los Biocarriers instalados en ambos tanques reactores biológicos. Los métodos de análisis de laboratorio fueron los mismos ya citados en la situación ex antes. Se midió el caudal efluente, hora a hora y por un periodo de tiempo de 17 horas, el 26/07/2015 se inició a las 00 horas, mientras que el 28/06/2015 se inició a las 05 horas.

\subsection{Técnica de análisis estadístico}

La herramienta o técnica que permite llevar a cabo este tipo de análisis es el denominado test de hipótesis, que se define de manera distinta en función del tipo de atributos con los que estemos trabajando. En función del tipo de atributo el presente trabajo de investigación ha usado la definición: Nominales-numéricos, es decir que, en este caso uno de los atributos toma valores de un conjunto de posibles valores y otra toma valores numéricos. La relación entre los atributos se obtiene mediante la comparación de medias y el análisis de varianza.

\section{RESULTADOS}

Para el desarrollo del monitoreo se consideraron los protocolos establecidos en los cuales se plantean los lineamientos básicos para su desarrollo, específicamente:

- $\quad$ RM N²73-2013-VIVIENDA: Protocolo de Monitoreo de Calidad de Efluentes de las Plantas de Tratamiento de Aguas Residuales Domésticas o Municipales.

- American Public Health Association (APHA, 2017). Standard Methods For the Examination of Water and Wastewater, 23nd edition

Las muestras obtenidas fueron analizadas en el laboratorio "ALS Corplab - Sede Arequipa" acreditado por INACAL. Las muestras de parámetros organolépticos, como $\mathrm{pH}$ y Temperatura, fueron tomados in situ. A continuación, se presentan los resultados de los monitoreos realizados en el afluente y efluente de las aguas residuales (ver Tabla 3).

Una vez regulado el ingreso de agua a la planta y ya estabilizada (alrededor de 2 meses) se pudo realizar el monitoreo de calidad del agua tratada, en la Tabla 3 se muestran los resultados de la toma de agua a la entrada de la PTAR observándose que los valores obtenidos son los correspondientes para un agua residual de tipo doméstica, siendo estos valores compatibles con el diseño de la PTAR para su tratamiento, mientras que en la Tabla 4, se muestran los resultados de la toma de agua a la salida de la PTAR, los cuales cumplen con el propósito de la investigación al encontrarse por debajo de los Límites Máximo Permisibles.

\section{DISCUSIÓN}

En la Tabla 5 se muestran los resultados de los aforos realizados antes de la instalación de los Biocarriers, esto con el fin de verificar que la calidad del efluente no se altera de manera negativa con el incremento de caudal de tratamiento hasta $280 \mathrm{~m}^{3} / \mathrm{d}$.

Se puede determinar que los resultados del análisis de laboratorio en cuanto a los parámetros de calidad del agua a la salida de la PTAR son equivalentes antes y después de la instalación de los Biocarriers, demostrándose la efectividad del sistema con un incremento del volumen a tratar del 40 $\%$ del total de la capacidad real de la planta.

Como referencia o antecedente de la investigación tenemos los aportes de (Sanabria Cubillos \& Ojeda, 2019) que en enero del 2019 realizaron el diseño y evaluación de un reactor biológico de lecho móvil de cargas secuenciales como alternativa de tratamiento para un vertimiento

Tabla 3. Resultados analíticos - entrada PTAR

\begin{tabular}{|c|c|c|c|c|}
\hline $\begin{array}{c}\text { Parámetro } \\
001 \text { Datos del Cliente }\end{array}$ & Ref. Mét. & Unidad & LD & Entrada PTAR \\
\hline $\mathrm{pH}^{*}$ & 2209 & Unidades pH & --- & 6,5 \\
\hline Temperatura de la Muestra & 2210 & ${ }^{\circ} \mathrm{C}$ & --- & 15,0 \\
\hline \multicolumn{5}{|l|}{003 ANALISIS FISICOQUIMICOS } \\
\hline Aceites y Grasas & 8833 & $\mathrm{mg} / \mathrm{L}$ & 1,0 & 17,9 \\
\hline Demanda Bioquímica de Oxígeno & 1828 & $\mathrm{mg} / \mathrm{L}$ & 2 & 159 \\
\hline Demanda Química de Oxígeno & 8803 & $\mathrm{mg} 02 / \mathrm{L}$ & 2 & 294 \\
\hline Fósforo Total & 8807 & $\mathrm{mg} P / \mathrm{L}$ & 0,012 & 4,373 \\
\hline Sólidos Totales Suspendidos & 1843 & $\mathrm{mg} / \mathrm{L}$ & 2 & 134 \\
\hline \multicolumn{5}{|l|}{014 ANALISIS MICROBIOLOGICOS } \\
\hline Coliformes Fecales & 8825 & $\mathrm{NMP} / 100 \mathrm{~mL}$ & 1,8 & 4600000 \\
\hline$N^{\circ}$ ALS - CORPLAB & & & & $193088 / 2015-1.0$ \\
\hline Fecha de muestreo & & & & $24 / 05 / 2015$ \\
\hline Hora de muestreo & & & & 12:30:00 \\
\hline Tipo de muestra & & & & Agua residual doméstica \\
\hline
\end{tabular}

Fuente. ALS - CORPLAB 
Tabla 4. Resultados analíticos - salida PTAR

\begin{tabular}{|c|c|c|c|c|c|}
\hline Datos & Unidad & LD & 01 & 02 & 03 \\
\hline Cloro residual/libre & $\mathrm{mg} / \mathrm{L}$ & -- & 1.00 & 1.00 & 1.00 \\
\hline $\mathrm{pH}$ & Unidades $\mathrm{pH}$ & -- & 8.00 & 8.00 & 8.00 \\
\hline Temperatura de la muestra & ${ }^{\circ} \mathrm{C}$ & -- & 17.00 & 18.00 & 17.00 \\
\hline Aceites y grasas & $\mathrm{mg} / \mathrm{L}$ & 1 & $<1.00$ & $<1.00$ & $<1.00$ \\
\hline DBO & $\mathrm{mg} / \mathrm{L}$ & 2 & 24.00 & 36.00 & 21.00 \\
\hline DQO & $\mathrm{mgO2/L}$ & 2 & 60.00 & 77.00 & 52.00 \\
\hline Fósforo total & $\mathrm{mg} \mathrm{P/L}$ & 0.012 & 2.16 & 2.74 & 2.01 \\
\hline Sólidos totales suspendidos & $\mathrm{mg} / \mathrm{L}$ & 2 & 17.00 & 21.00 & 16.00 \\
\hline Coliformes fecales & $\mathrm{NMP} / 100 \mathrm{ml}$ & 1.8 & 1400 & 2100 & 1200 \\
\hline Fecha de muestreo & -- & -- & $24 / 05 / 2015$ & $28 / 06 / 2015$ & $26 / 07 / 2015$ \\
\hline Hora de muestreo & -- & -- & $12: 55: 00$ & $11: 45: 00$ & 13:00:00 \\
\hline Tipo de muestra & -- & -- & $\begin{array}{l}\text { Agua residual } \\
\text { doméstica }\end{array}$ & $\begin{array}{l}\text { Agua residual } \\
\text { doméstica }\end{array}$ & $\begin{array}{l}\text { Agua residual } \\
\text { doméstica }\end{array}$ \\
\hline Identificación & -- & -- & Efluente & Efluente & Efluente \\
\hline Parámetro & -- & -- & -- & -- & -- \\
\hline
\end{tabular}

Fuente. ALS - CORPLAB

Tabla 5. Características de las aguas afluente y efluente

\begin{tabular}{|c|c|c|c|c|c|}
\hline Parámetros & Unidades & LMP $\left(^{*}\right)$ & Afluente & Efluente & \% Remoción \\
\hline pH & Adm. & $6.5-8.5$ & 6.5 & 8 & - \\
\hline Temperatura & ${ }^{\circ} \mathrm{C}$ & $<35$ & 15 & 17 & - \\
\hline Aceites y grasas & $\mathrm{mg} / \mathrm{L}$ & 20 & 17.9 & $<1$ & - \\
\hline $\mathrm{DBO}_{5}$ & $\mathrm{mg} / \mathrm{L}$ & 100 & 159 & 24 & $84.91 \%$ \\
\hline DQO & $\mathrm{mg} / \mathrm{L}$ & 200 & 294 & 60 & $79.59 \%$ \\
\hline Sólidos Suspendidos Totales & $\mathrm{mg} / \mathrm{L}$ & 150 & 134 & 17 & $87.31 \%$ \\
\hline Coliformes Fecales-CF- ó Termotolerantes & $\mathrm{NMP} / 100 \mathrm{ml}$ & 10000 & 4600000 & 1400 & $99.97 \%$ \\
\hline
\end{tabular}

Fuente. ALS - CORPLAB

(*) DS N003-2010-MINAM: LMP para efluentes de PTAR de origen doméstico

procedente de una industria farmacéutica. Como resultado de esta investigación el reactor alcanzo eficiencias de remoción de DQO de hasta $92 \%$, sin embargo, se atribuye dicha disminución a la biomasa que se encontraba en suspensión ya que en los portadores plásticos no se logró percibir cualitativamente en ningún momento la adherencia de la biomasa al portador. La falencia principal es que se diseñó con requerimientos de oxígeno de un reactor convencional de lodos activados, lo que puede tener incidencia en la cantidad de aire que realmente requiere puesto que su operación es por adherencia de biomasa en un portador.

Otro referente es la investigación realizada por (Parra Riquelme, s. f.) en diciembre del 2006 - Optimización de un biorreactor de lecho móvil (MBBR) para la biodegradación de un efluente proveniente de la industria de celulosa Kraft. Como resultado de esta investigación se obtuvo que las mejores eficiencias de depuración fueron a un TRH (tiempo de retención hidráulica) de 0,9 días, mostrando porcentajes de remoción para DQO superiores al $45 \%$ y para la $\mathrm{DBO}_{5}$ por sobre el 97\%. Haciendo una comparación en referencia a los resultados de nuestra investigación podemos concluir que nuestros porcentajes de remoción de DQO y DBO son mucho más homogéneos y contrastan de manera sostenible durante el proceso. A continuación, se muestra una imagen referencial del funcionamiento del sistema MBBR: (Ver figura 1).

\section{CONCLUSIONES}

- La adecuación del sistema MBBR de la planta de tratamiento de aguas residuales de origen doméstico de Caylloma, del tipo lodos activados, permite tratar un $40 \%$ más de la capacidad nominal de los tanques reactores, en este caso de 200 a $280 \mathrm{~m} 3 / \mathrm{d}$, sin alterar la calidad del efluente de la planta, por lo tanto: 
Reactor biológico MBBR Decantador secundario

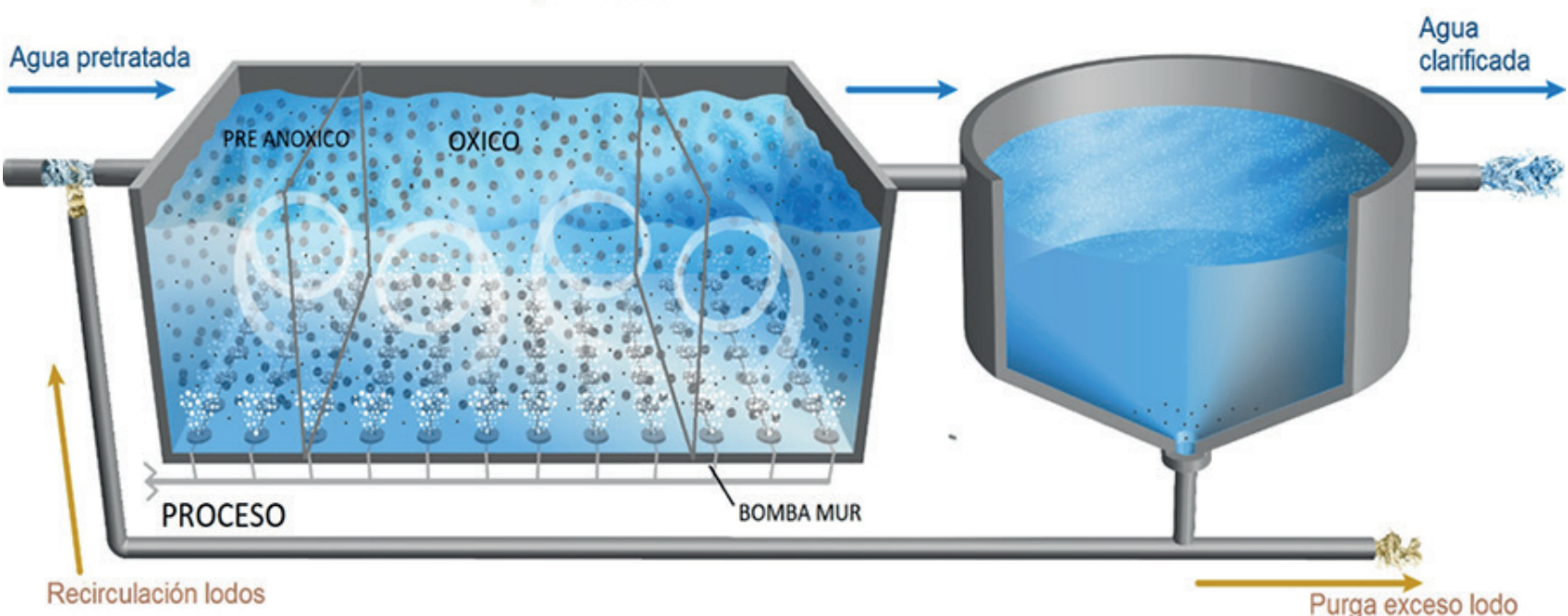

Figura 1. Esquema de funcionamiento del sistema MBBR. Indiamart - Artículo

- Permite menores tiempos de retención hidráulica, lo cual se traduce en una mayor cantidad de agua tratada en menor tiempo.

- La adición de los Biocarriers influye sobre el comportamiento biológico de los procesos de degradación de materia orgánica, aumentando la estabilización del sistema debido al incremento de la concentración de la biomasa en el reactor biológico (Pascual, 2014). Esto acelera el proceso de tratamiento y resulta en la obtención de un efluente cuya calidad es óptima para ser usado en el riego de vías, caminos o áreas verdes.

- La calidad final del efluente a la salida de la PTAR con la implementación del sistema MBBR, en comparación con la calidad del efluente sin la implementación de este sistema se mantiene equivalente. Esto demuestra la efectividad del sistema MBBR, que posibilita utilizar el efluente para fines de riego mediante la aplicación de pequeños ajustes.

- El sistema genera costos de implementación mínimos, los costos de inversión solo requirieron la adquisición de los Biocarriers; los costos de operación y mantenimiento se incrementan mínimamente por la cantidad insumos químicos necesarios.

- Como se ha demostrado, incorporar a los tanques biorreactores los "Biocarriers" constituye una tecnología novedosa en nuestro país, donde predominan las unidades de tratamiento tipo lagunas facultativas $(78 \%$ de 112 PTAR), tal como se señala en: Diagnostico Situacional de los Sistemas de Tratamiento de Aguas Residuales en las EPS del Perú y Propuestas de Solución (SUNASS, s. f.), donde se resalta como principal causa de tratamiento inadecuado de las aguas residuales domésticas, la insuficiencia de investigaciones y desarrollo tecnológico en el Perú.

- Con el sistema MBBR se optimizan los procesos de tratamiento en los biorreactores, lo que permite obtener que la calidad del efluente cumpla con la normatividad vigente no alterando por ende el ecosistema donde finalmente son vertidos.

\section{AGRADECIMIENTOS}

Mi especial agradecimiento al Ing. Alberto Quenaya gerente general de Aquafil - Wáter Technologies, por su asesoría y apoyo incondicional en la realización de esta tesis, además de su preocupación e interés en la mejora continua y avances tecnológicos.

De igual forma debo agradecer a San Martín Contratistas Generales S.A. en nombre del ing. Juan José Gambini, Gerente Corporativo de Seguridad, Salud Ocupacional y Medio Ambiente de esta importante empresa, por permitirme realizar este trabajo de investigación brindándome todas las facilidades para su desarrollo.

\section{REFERENCIAS}

APHA. (2017). Standard Methods For the Examination of Water and Wastewater, 23nd edition. Standard Methods For the Examination of Water and Wastewater, 23nd Edition. https:// www.academia.edu/38769108/Standard Methods For the Examination of Water and Wastewater 23nd edition

Parra Riquelme, C. P. (s. f.). Optimización de un biorreactor de lecho móvil (MBBR) para la biodegradación de un efluente proveniente de la industria de celulosa Kraft. 44. 
Pascual, J. M. (2014). Estudio técnico de biorreactores de membrana con lecho móvil aplicados al tratamiento de aguas residuales (p. 1) [Http://purl.org/dc/dcmitype/Text, Universidad de Granada]. https://dialnet.unirioja.es/servlet/ tesis? codigo $=58550$

Sanabria Cubillos, A., \& Ojeda, J. P. (2019). Diseño y evaluación de un reactor biológico de lecho móvil de cargas secuenciales como alternativa de tratamiento para un vertimiento procedente de una industria farmacéutica. Ingeniería Ambiental y Sanitaria. https://ciencia.lasalle.edu. co/ing_ambiental_sanitaria/780
SUNASS. (s. f.). Recuperado 7 de junio de 2020, de https://www. sunass.gob.pe/doc/Publicaciones/libro_ptar_gtz_sunass.pdf

Wanner, O., \& Reichert, P. (1996). Mathematical modeling of mixed-culture biofilms. Biotechnology and Bioengineering, 49(2), 172-184. https://doi.org/10.1002/(SICI)10970290(19960120)49:2<172::AID-BIT6>3.0.CO;2-N 
